\title{
Efficacy of urination in alleviating man's urethral pain associated with flexible cystoscopy: a single-center randomized trial
}

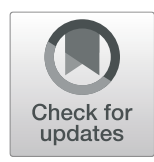

\author{
Yingwei Xie, Wei Wang, Wei Yan, Dan Liu and Yuexin Liu* (1)
}

\begin{abstract}
Background: This study aimed to assess whether urethral pain can be alleviated by urination in male patients undergoing flexible cystoscopy.

Methods: Ninety-six male outpatients undergoing flexible cystoscopy were randomly divided into two groups. Patients in the test group urinated during flexible cystoscopy, whilst patients in the control group received no instructions to do so. All patients received $10 \mathrm{~mL}$ of $2 \%$ lidocaine gel prior to assessment. Using 0 (no-pain) to 10 (unbearable severe pain) pain scores (VAS), we assessed patient discomfort prior to anesthesia gel perfusion (baseline), during gel perfusion, during cystoscope insertion through the urethra, and 15 min post-examination analysis. The entire protocol was completed by a single doctor in our Department of Urology.
\end{abstract}

Results: The groups showed no statistical differences regarding age or examination time. During cystoscope insertion, the test group recorded significantly lower pain scores 2 (IQR 1-3) - compared to the control group 3 (IQR 2-3), $(P=0.001)$. No significant differences between other evaluation points were observed between groups.

Conclusion: Urethral pain can be significantly alleviated by urination in male patients undergoing flexible cystoscopy through the urethra.

Trial registration: Registry name: Clinical study of urination action to relieve urethral pain associated with flexible cystoscopy.

Registration number: ChiCTR-INR-17013294

Date of Registration: 2017-11-08

Keywords: Flexible cystoscopy, Bladder, Pain

\section{Background}

Cystoscopy is one of the most important tools for disease diagnosis in urology. Since its inception in 1984, flexible cystoscopy has been the procedure of choice for this purpose. Compared to rigid cystoscopes, flexible cystoscopy has the advantage of no blind areas, small damage, clear vision and a low requirement for body positioning. During analysis, the cystoscope must pass through the urethra to reach the bladder resulting in contact with the urethra mucosal membranes, leading to discomfort and pain. Patients receiving this procedure

\footnotetext{
* Correspondence: docxyx@126.com

Department of Urology, Beijing Tongren Hospital, Capital Medical University, Beijing 100730, China
}

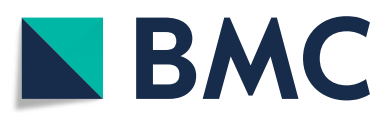

(c) The Author(s). 2020 Open Access This article is distributed under the terms of the Creative Commons Attribution 4.0 International License (http://creativecommons.org/licenses/by/4.0/), which permits unrestricted use, distribution, and reproduction in any medium, provided you give appropriate credit to the original author(s) and the source, provide a link to the Creative Commons license, and indicate if changes were made. The Creative Commons Public Domain Dedication waiver (http://creativecommons.org/publicdomain/zero/1.0/) applies to the data made available in this article, unless otherwise stated.

are thus often stressed, have increased heart rates and elevated blood pressure $[1,2]$.

Methods to alleviate pain in patients undergoing flexible cystoscopy have been widely investigated. Local anesthesia is a widely used analgesic method in flexible cystoscopy. Its analgesic theory is to inject anesthetic and penetrating drugs into the urethra to block the nerve located under the mucosa, thus playing the role of anesthesia. Local anesthetics are mostly gelatinous mixtures of anesthetics and lubricants, such as lidocaine gel, bupivacaine gel, tetracaine gel, etc. These anesthetic gels can not only play an anesthetic role in the urethra but also have lubrication. Lidocaine is currently the most widely used local anesthetic. However, recent studies have shown that lidocaine gel has no obvious advantage 
over urethra pain control compared with ordinary gel, and may cause additional pain and discomfort [3-5]. In addition to the use of anesthetics to relieve pain, more and more attention has been paid to the study of nondrug analgesic methods in recent years. There are many studies to alleviate pain through psychological or behavioral interventions. For example, watching the examination process through closed-circuit television $[6,7]$, listening to music $[8,9]$, etc.

We believe that defining the specific site of urethral pain caused by cystoscopy can help us control the pain more effectively. Chen [10] and Taghizadeh [11] have demonstrated that the most painful part of flexible cystoscopy is when the tip of the cystoscope passes through the external sphincter. Anatomically, the external urethral sphincter is located in the urethral membrane of the urethral stricture, and is also the most abundant area of afferent nerves. During urine storage, the external urethral sphincter is in a state of contraction and closes the membranous urethra. During urine passage, the external urethral sphincter relaxes [12].

We thus assumed that during the course of flexible cystoscopy, if the patient is urinating according to defined instructions, the external sphincter can relax, thereby reducing urethral pain during cystoscope insertion. Based on this hypothesis, we designed a prospective, randomized, controlled trial in which we assessed the levels of pain in patients instructed to urinate during flexible cystoscopy.

\section{Methods}

\section{Ethical approval and trial registration}

The trial was conducted between December 2017 and April 2018 in the Beijing Tongren Hospital in Beijing, China. The study was approved by the ethics committee of the Beijing Tongren Hospital, Capital Medical University (approval number: TRECKY2017-034) and registered at chictr.org.cn (registry number: ChiCTR-INR17013294, date of registration 08 November 2017), prior to patient recruitment. The study was conducted according to common standard guidelines for clinical trials (Declaration of Helsinki, and the International Conference on Harmonization of Technical Requirements for Registration of Pharmaceuticals for Human Use and Good Clinical Practice (ICH-GCP) revised version, Somerset West, Republic of South Africa, 1996) [13]. All patients involved in this study gave their informed consent.

\section{Patients}

Male patients were admitted to our clinic for flexible cystoscopy. All patients were over 18 years old. Exclusion criteria included any analgesic used within $24 \mathrm{~h}$ of the study period, known urethral stricture, previous history of urethral dilation, acute urinary tract infection, indwelling urethral catheter, and existing urethral pain (including chronic pelvic inflammatory disease and interstitial cystitis) Patients, as well as those who are unable to cope with pain assessment due to mental disorders.

\section{Randomization}

Patients were screened by the chief physician of the outpatient clinic. Use a computer (Random Allocation Software, version 1.0.0) to build a random number table, then randomly divide patients into two groups. The randomisation was carried out by an independent researcher who was not involved in the subjectrecruitment process. The sealed opaque envelopes were used to ensure concealed allocation. Intervention participants in the trial knew the patient's grouping, and information collectors did not know the patient's grouping.

\section{Design}

We performed a prospective, randomized, controlled trial designed to compare pain scores between patients in experimental- and controls groups. Patients in the test group urinated during flexible cystoscopy. Patients in the control group received no instructions during the procedure. During examination, $10 \mathrm{mls}$ of $2 \%$ lidocaine gel was injected into the urethra and the penis was clamped for $10 \mathrm{~min}$. A flexible cystoscope was passed through the urethra into the bladder for examination. All cystoscopies were performed by an experienced urologist. Flexible cystoscopy instruments included digital camera (Olympus OTV-S7), color video monitor (Olympus OEV-191H) and 16F flexible cystoscope (Olympus CYF-5A).

\section{Intervention}

The patient was taken supine position during cystoscopy. Routine disinfection. During examination, $10 \mathrm{mls}$ of $2 \%$ lidocaine gel was injected into the urethra and the penis was clamped for $10 \mathrm{~min}$. The procedure of the control group was as follows: the examiner lifted the penis with his left hand, inserted the front end of the flexible cystoscope into the urethra with the help of his left thumb and index finger, and held the mirror with his right hand. The thumb controlled the adjusting lever. With the help of water flow, the examiner observed the urethral side entry mirror under video surveillance. Stay $1-2 \mathrm{~s}$ when seeing the external urethral sphincter and entered the bladder under direct vision. In the experimental group, the examiner lifts the penis with his left hand, inserts the front end of the flexible cystoscope into the urethra with the help of his left thumb and index finger, holds the mirror with his right hand, and controls the control lever with his thumb. With the help of water flow, the examiner observed the urethral side entry 
mirror under video surveillance. Stay for 1-2 s when seeing the external urethral sphincter and ask the patient to urinate. Then entered the bladder under direct vision.

\section{Assessment}

Patient histories were collected prior to operation. During cystoscopy, an experienced urologist nurse recorded the pain score according to the patient's assessment before aesthesia gel perfusion (baseline), anaesthetized gel perfusion, cystoscopy insertion into the urethra, and 15 min post-examination analysis. Patients were asked to cross a line in a $10 \mathrm{~cm}$ region with 0 at one end of the transverse line indicating no pain; and 10 at the other end indicating a sharp intense pain.

\section{Collection of co-variate data}

Before the experiment, researchers collected general information about patients, including age, height, weight, and underlying diseases.

\section{Sample size calculation}

We used the result of Taghizadeh as the pain score of our control group, $2.82 \pm 1.2$ VAS [11]. A minimal clinically important difference according to Todd was 1.3 VAS [14]. Given the effect size of Cohen's $d=0.615$, and a two-sided $5 \%$ level t-test with a statistical power of 1$\beta=80 \%$, 43 patients would be needed to detect this group difference. We planned to include 96 patients in this trial ( $n=48$ per group); recognizing a potential loss of analytical power due to patient withdrawal from $10 \%$.

\section{Data analyses}

SPSS20.0 software was used for all data analysis. Data are expressed as mean \pm standard deviation (SD), median and interquartile range (IQR) for variables that do not follow a normal distribution, or frequencies for categorical data. Normally distributed continuous variables were compared between the two groups using a two-sided ttest, with chi-square tests for categorical variables and with non-parametric Wilcoxon test for not normally distributed continuous variables. A $p$-value less than 0.05 was considered to be statistically significant.

\section{Results}

The trial included 96 patients (48 cases in each group). As shown in Table 1, the average age of the test group was $53.35 \pm 15.87$ years, whilst the control group had an average age of $54.10 \pm 15.48$ years, which did not significantly differ $(P=0.815)$. Study groups did not differ in demographics, co-morbidities. The mean examination times were $3.42 \pm 0.64$ mins for the test group and $3.54 \pm 0.71$ mins for the control group, which did not significantly differ $(P=0.371)$.
Table 1 Patient data obtained during the study protocol

\begin{tabular}{llll}
\hline & $\begin{array}{l}\text { Test group } \\
(n=48)\end{array}$ & $\begin{array}{l}\text { Test group } \\
(n=48)\end{array}$ & $P$ value \\
\hline Years $^{\text {a }}$ & $53.35 \pm 15.87$ & $54.10 \pm 15.48$ & 0.815 \\
Mean Body mass index $^{\mathrm{a}}$ & $26 \pm 3$ & $26 \pm 3$ & 0.142 \\
Hypertension n (\%) & $15(31)$ & $13(27)$ & 0.156 \\
Diabetes Mellitus n (\%) & $7(15)$ & $11(23)$ & 0.122 \\
Examination times (min) $^{\mathrm{a}}$ & $3.42 \pm 0.64$ & $3.54 \pm 0.71$ & 0.371 \\
\hline
\end{tabular}

${ }^{a}$ Data presented as mean \pm SD for normally distributed data or as percentage for categorical data

Regarding the VAS scores, no obvious urethral pain discomfort prior to anesthesia gel perfusion (baseline) was observed in either group (all scores $=0$ ). When the two groups were compared after gel perfusion and 15 min following examination, no significant differences were observed $(P=0.761 \& P=0.661$, respectively). Upon comparison of the scoring when the cystoscope was inserted, the score of the test group was 2 (IQR 13) compared to 3 (IQR 2-3) for the control group, which was significantly lower $(P=0.001)$ (Fig. 1$)$.

\section{Discussion}

Cystoscopy is routinely performed during urology assessments, particularly following bladder cancer surgery. It is therefore important to minimize the pain caused by this examination procedure. The invention of a flexible cystoscope greatly reduced the discomfort experienced during a patient's examination and improved patient tolerance. However, pain was still unavoidable. Topical local anesthesia is currently widely employed but no consensus on the dosage, temperature, onset time and efficacy of its use have been assessed $[4,15]$. In addition to the use of lubricants, more attention has been paid to non-pharmacological analgesic methods in recent years, including psychological and behavioral interventions. Patel \& Zhang demonstrated that watching the examination process through closed-circuit television relieves urethral pain $[6,7]$, but these findings were refuted in later studies [16, 17]. Gunendran and coworkers found that squeezing brine bags significantly reduced pain and recommended the routine application of this procedure in males [18]. Zhang \& Raheem also demonstrated that when patients listened to music, reduced pain and anxiety were observed $[8,9]$.

Due to the different urethral anatomy characteristics of male and female patients, the location and degree of pain caused by cystoscopy differs. When cystoscopy is performed, obvious resistance in the male urethra is sensed, particularly when the catheter passes through the urethral membrane. Taghizadeh and colleagues performed assessments in male patients in which they allowed patients to squeeze a balloon filled with water during contact with various areas of the urethra, 


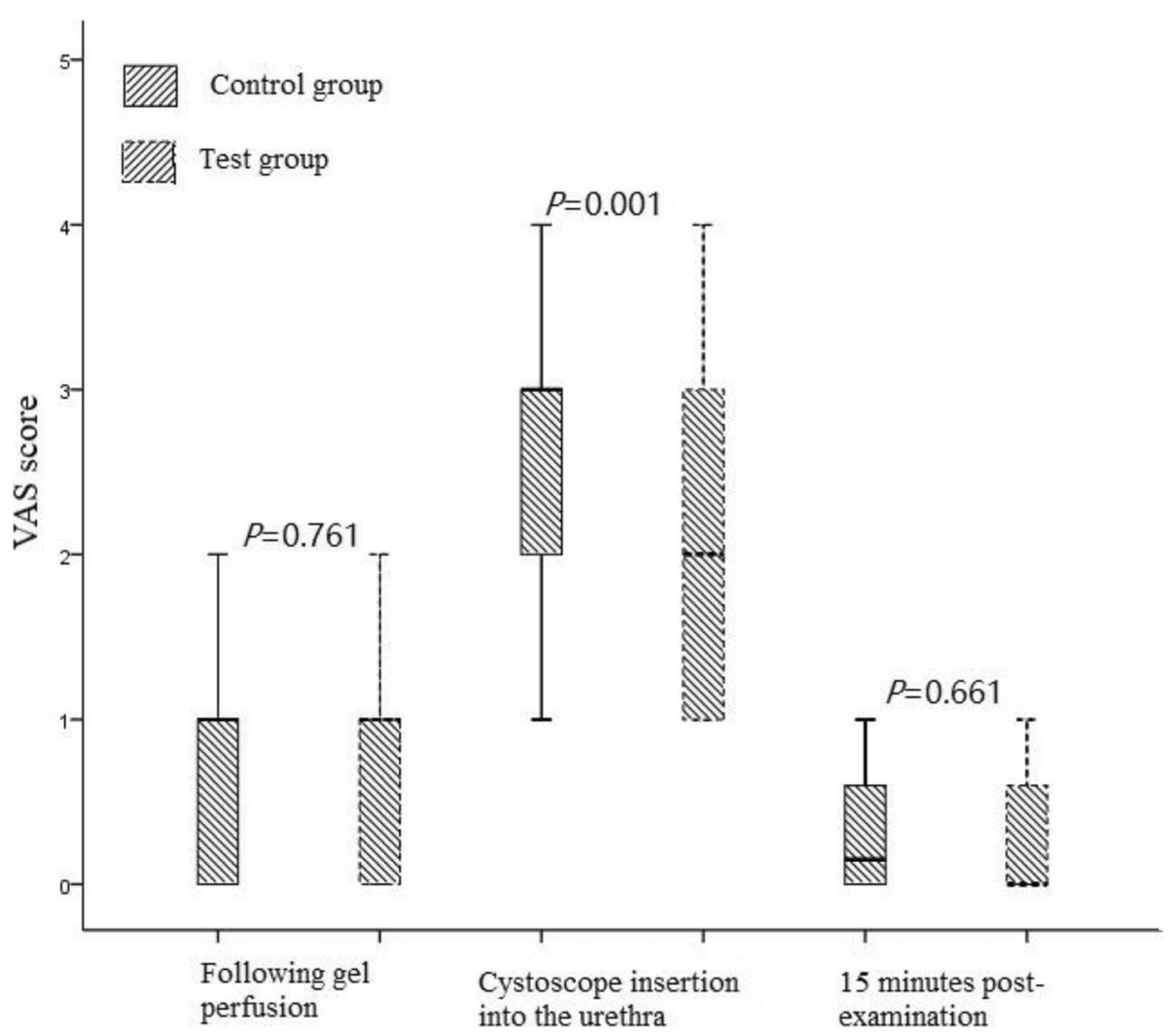

Fig. 1 Comparison of the pain scores between the 2 groups at different checkpoints during cystoscopy

evaluating the patient pain by the levels of water ejected. The results showed that cystoscopy caused the most significant pain when contacting the urethral membrane, where an average pain score of 2.82 was recorded. The second highest pain score occurred when the anaesthetized gel was squeezed into the urethra, displaying an average pain score of 0.84 [11]. This study provided direction for the future assessment of new analgesic methods for cystoscopy. Methods to reduce the pain caused by the flexible cystoscope passing through the urethral membrane appeared the key aspect.

In the above studies, patients were allowed to undertake deep breathing, observe closed-circuit television, and listen to music, in essence, to relax the external sphincter and reduce friction of the urethra membrane. The same effect is achieved by squeezing saline bags. In this study, we reasoned that further methods to relax the external urethral sphincter may further relieve the pain and discomfort experienced by patients. Anatomically, the urethral membrane is surrounded by an external urethral sphincter belonging to the striated muscle, which functions to control urination. In urodynamic studies, the external urethral sphincter is continually contracting during normal urine storage. At the beginning of urination, the external urethral sphincter relaxes, the urethral pressure drops, the bladder pressure rises, and urine is expelled from the urethra. Adults with normal nervous system functionality can initiate or suppress urination at will, even when the bladder contains only small amounts of urine. The cerebral cortex can also relieve inhibition of the urination center of the pons and urinate [19]. This provided a theoretical basis for this study. The maximum urethral pressure in urodynamics is equivalent to the urethral pressure at the external sphincter of the urethra. The maximum urethral pressure during adult male urine storage is $72.70+5.07 \mathrm{cmH} 2 \mathrm{O}$, whilst the pressure of the external urethral sphincter decreases to $25-30 \mathrm{~cm} \mathrm{H} 2 \mathrm{O}$ during urination [12]. This provides a clinical basis for this study. The ability to actively control the cerebral cortex through active urination intention leads to relaxation of the external urinary sphincter and would allow the cystoscope to pass more smoothly. In our trial, we demonstrated that the VAS score among male patients who urinated during flexible cystoscopy was significantly lower. The mean VAS score had a decrease from 3 to 2. Whilst this study, we did not observe obvious relaxation of external urethral sphincter, the patient pain scores were significantly lower than those of the control group. In was likely that patients were attempting to prevent voluntary or reflex activation of the striated sphincter that occurs as a response to apprehension of catheterization and/or as a reflex response to 
afferent pudendal reflex arc stimulation, compared to if the reflex relaxation typically occurred without detrusor activation.

Thus, we have demonstrated that urination during flexible cystoscopy can significantly reduce cystoscopy induced pain in the urethra. Similarly, Stav K permits patients to perform urination during male catheterization, which contributes to relieving urethral pain [20].

In this study, the patient's pain score decreased from 3 (IQR 2-3) to 2 (IQR 1-3), $P=0.001$. Explain that our experiments have worked. However, from the clinical point of view, the extent of pain reduction is still small, and the pain score of cystoscopy itself is not high, so the clinical significance is small. We believe that this test can be used in rigid cystoscopy which can causes severe pain in the future to evaluate the clinical effect of this method.

This study has the following advantages: In addition to prospective, random controls, all operations were performed by the same highly skilled physician, thus maintaining consistency. Secondly, the characteristics of the two study groups were similar and both groups underwent comparable examination times. Thus, bias and other confounding factors were reduced to a minimum. The limitations of this study included the uncertainty that patients in the control group displayed no autonomous urination. In addition, apart from the subjective pain score, we had no objective evidence that the external urethral sphincter relaxed. Future studies should employ external urinary sphincter electromyography to ensure an objective evaluation of our study findings.

\section{Conclusions}

In summary, we suggest that during male cystoscopy, patient urination represents a means of reducing pain levels during the examination.

\section{Abbreviation}

VAS: Visual analogue scale

\footnotetext{
Acknowledgements

I am very grateful to my supervisor, Professor Liu Yuexin, for his guidance and assistance in the whole research process. At the same time, I want to thank Professor Wang Wei and Professor Liu Dan for monitoring and advices on my research process. I would also like to thank Professor Yan Wei, who has a lot of experience in cystoscopy, for playing a crucial role in my entire study. Finally, I would like to thank other colleagues and teachers in Department of Urology for their help.
}

\section{Authors' contributions}

YX wrote the main manuscript text and prepared all figures. YL and WW supervised the research and helped write the manuscript. WY was responsible for the operation of cystoscopy in the experiment. $D L$ is responsible for collecting and processing data. All authors have read and approved the manuscript.

\section{Funding}

No funding was obtained for this study.
Availability of data and materials

The datasets during and/or analyzed during the current study available from the corresponding author on reasonable request.

\section{Ethics approval and consent to participate}

The trial was conducted between December 2017 and April 2018 in the Beijing Tongren Hospital in Beijing, China. The study was approved by the ethics committee of the Beijing Tongren Hospital, Capital Medical University (approval number: TRECKY2017-034) and registered at chictr.org.cn (registry number: ChiCTR-INR-17013294, date of registration 08 November 2017), prior to patient recruitment. Each patient signed an informed consent before the trial.

\section{Consent for publication}

All of the details can be published and consent for publication was not required for this study.

\section{Competing interests}

The authors declare that they have no competing interests.

Received: 10 June 2019 Accepted: 21 October 2019

Published online: 20 January 2020

\section{References}

1. Pillai PL, Sooriakumaran P. Flexible cystoscopy: a revolution in urological practice. Br J Hosp Med. 2009;70:583-5.

2. Wojciech $\mathrm{K}$, et al. Patient comfort during flexible and rigid cystourethroscopy. Wideochir Inne Tech Maloinwazyjne. 2016;11:94.

3. Akkoç A, et al. $2 \%$ Lidocaine gel or plain lubricating gel: Which one should be used in male flexible cystoscopy? Turk J Urol. 2016:42:92.

4. Patel AR, Jones JS, Babineau D. Lidocaine $2 \%$ gel versus plain lubricating gel for pain reduction during flexible cystoscopy: a meta-analysis of prospective, randomized, controlled trials. J Urol. 2008;179:986-90.

5. Ho KJ, Thompson TJ, O'Brien A, Young MRA, Mccleane G. Lignocaine gel: does it cause urethral pain rather than prevent it? Eur Urol. 2003;43:194-6.

6. Patel AR, Jones JS, Angie S, Babineau D. Office based flexible cystoscopy may be less painful for men allowed to view the procedure. J Urol. 2007; 177:1843-5.

7. Zhang ZS, Tang L, Wang $X L$, Xu CL, Sun YH. Seeing is believing: a randomized controlled study from China of real-time visualization of flexible cystoscopy to improve male patient comfort. J Endourol. 2011;25:1343-6.

8. Zhang ZS, et al. Music reduces panic: an initial study of listening to preferred music improves male patient discomfort and anxiety during flexible cystoscopy. J Endourol. 2014;28:739-44.

9. Raheem $\mathrm{O}$, et al. Does listening to music during office-based flexible cystoscopy decrease anxiety in patients: a prospective randomized trial. J Urol. 2016:195:448-9.

10. Chen YT, et al. Randomized double-blind comparison of lidocaine gel and plain lubricating gel in relieving pain during flexible cystoscopy. J Endourol. 2005;19:163-6.

11. Taghizadeh AK, et al. When does it hurt? Pain during flexible cystoscopy in men. Urol Int. 2006;76:301-3.

12. Xiyu J, Bo S. In: Bo S, editor. Clinical urodynamics. Beijing: People's Health Publishing House; 2002. p. 76-7. Chinese.

13. Inc W M A. Declaration of Helsinki. Ethical principles for medical research involving human subjects. J Indian Med Assoc. 2009;14(1):233-8.

14. Todd KH, Funk KG, Funk JP, Bonacci R. Clinical significance of reported changes in pain severity. Ann Emerg Med. 1996:27:485-9.

15. Aaronson DS. Meta-analysis: does lidocaine gel before flexible cystoscopy provide pain relief? J Urol. 2009;104:8,

16. Koenig J, et al. Does visualisation during urethrocystoscopy provide pain relief? Results of an observational study. BMC Urol. 2015;15:1-6.

17. Cornel EB, Oosterwijk E, Kiemeney LA. The effect on pain experienced by male patients of watching their office-based flexible cystoscopy. BJU Int. 2008:102:1445-6.

18. Jones JS. Does increasing hydrostatic pressure ("bag squeeze") during flexible cystoscopy improve patient comfort: a randomized, controlled study - Comment. Urology. 2008;72:258

19. Blok BF, Willemsen AT, Holstege G. A PET study on brain control of micturition in humans. Brain. 1997;120:111. 
20. Stav K, Rappaport YH, Beberashvili I, Zisman A. Pain associated with urethral catheterization is reduced in males by simultaneous voiding maneuver. Urology. 2017;102:21-5.

\section{Publisher's Note}

Springer Nature remains neutral with regard to jurisdictional claims in published maps and institutional affiliations.

Ready to submit your research? Choose BMC and benefit from:

- fast, convenient online submission

- thorough peer review by experienced researchers in your field

- rapid publication on acceptance

- support for research data, including large and complex data types

- gold Open Access which fosters wider collaboration and increased citations

- maximum visibility for your research: over $100 \mathrm{M}$ website views per year

At BMC, research is always in progress. 